\title{
Embolia por proyectil por arma de fuego: a propósito de un caso
}

\author{
Pulmonary projectile embolism by firearm: A case report
}

\author{
Mateo Zuluaga $\mathbb{D}^{\mathbb{D}}$, Andrés Felipe Estrada ${ }^{2} \mathbb{D}$, María Claudia Rojas ${ }^{3} \mathbb{D}$, María Claudia Uribe ${ }^{4} \mathbb{D}$ \\ 1 Médico, residente en Medicina de Urgencias, Universidad CES; estudiante de la especialización en Gerencia de IPS, Universidad \\ CES; línea de investigación en Medicina de Urgencias y Toxicología CES, Medellín, Colombia \\ 2 Médico, especialista en Medicina de Urgencias, Hospital Pablo Tobón Uribe; especialista en Gerencia de IPS; docente de cáte- \\ dra, Facultad de Medicina, Universidad Pontificia Bolivariana; línea de investigación Medicina de Urgencias y Toxicología CES, \\ Medellín, Colombia \\ 3 Médica, especialista en Medicina de Urgencias, Hospital Pablo Tobón Uribe, especialista en Gerencia de IPS, Universidad CES, \\ Medellín, Colombia \\ 4 Médica, especialista en Medicina de Urgencias, Hospital Pablo Tobón Uribe, Medellín, Colombia
}

\section{Resumen}

Introducción. La embolia pulmonar por herida con arma de fuego es potencialmente fatal y una complicación rara que puede presentarse en los servicios de urgencias. En los últimos años, se ha presentado una mayor incidencia por la violencia social civil (85\%) que por las guerras (15\%). Los principales síntomas de los pacientes son dolor torácico, disnea y hemoptisis por la erosión vascular y el infarto pulmonar asociado.

Caso clínico. Se presenta el caso de un paciente de i8 años de edad que ingresó por múltiples heridas por proyectiles de arma de fuego, una de ellas con un proyectil alojado en la arteria pulmonar del lóbulo inferior derecho y embolia pulmonar aguda en las ramas arteriales distales al proyectil. Se optó por un tratamiento conservador y la evolución fue adecuada.

Discusión. La sintomatología de esta condición depende de la localización, la trombosis asociada, los fenómenos de isquemia y las complicaciones hemorrágicas. Entre las complicaciones de un proyectil de fuego alojado en una arteria pulmonar, están la isquemia, la trombosis, los pseudoaneurismas, la hemorragia, el derrame pleural, la insuficiencia vascular y la endocarditis. La mayoría de los pacientes deben someterse a una intervención quirúrgica cuando el proyectil se aloja en las arterias pulmonares principales o lobares y las compromete; cuando se localiza en las arterias segmentarias y subsegmentarias, tienden a presentar fístulas con el bronquio y vasoespasmo compensatorio, lo que permitiría el manejo conservador.

Palabras clave: lesiones del sistema vascular; heridas por arma de fuego; embolia; embolia pulmonar; tratamiento conservador.

Fecha de recibido: 20/06/2019 - Fecha de aceptación: 17/10/2019

Correspondencia: Mateo Zuluaga Gómez, Carrera 27 N³8B-Sur-10, apartamento 808, Edificio Escarlata, código postal 055420 Envigado, Colombia. Teléfono: (54) (313) 679-0511

Correo electrónico: mateozg92@hotmail.com

Citar como: Zuluaga M, Estrada AF, Rojas MC, Uribe MC. Embolia por proyectil por arma de fuego: a propósito de un caso. Rev Colomb Cir. 2020;35:514-9. https://doi.org/10.30944/20117582.731

Este es un artículo de acceso abierto bajo una Licencia Creative Commons - BY-NC-ND https://creativecommons.org/licenses/by-ncnd/4.0/deed.es 


\begin{abstract}
Introduction: Gunshot wound pulmonary embolism is potentially fatal and a rare complication that can occur in emergency departments. In recent years, there has been a higher incidence of civil social violence ( $85 \%$ ) than for wars (15\%). The main symptoms of patients are chest pain, dyspnoea and hemoptisis from vascular erosion and associated pulmonary infarction.

Clinical case: We present a case of an I8-year-old patient who was admitted for multiple projectile wounds by firearm, one of them with a projectile lodged in the pulmonary artery of the right lower lobe and acute pulmonary embolism in the arterial branches distal to the projectile. Conservative management was decided, with adequate evolution.

Discussion: The symptomatology of this condition depends on the location, associated thrombosis, ischemia phenomena and hemorrhagic complications. Complications of a fire projectile lodged in a pulmonary artery include ischemia, thrombosis, pseudoaneurysms, bleeding, pleural effusion, vascular insufficiency, and endocarditis. Most patients must undergo surgery when the projectile is housed in the main pulmonary arteries or lobar, when located in the segmental and subsegmental arteries tend to have fistulas with bronchi and compensatory vasospasm, which would allow conservative management.
\end{abstract}

Key words: vascular system injuries; wounds, gunshot; embolism; pulmonary embolism; conservative treatment.

\section{Introducción}

La embolia pulmonar por herida por arma de fuego es potencialmente fatal y una complicación rara que puede presentarse en los servicios de urgencias I. Clásicamente, se han reportado casos en los ambientes de guerra, pero en los últimos años, se ha presentado una mayor incidencia por la violencia social civil. Los síntomas son variables, desde disnea, hemoptisis o dolor torácico, hasta inestabilidad hemodinámica.

Se presenta un caso inusual de embolia pulmonar secundaria a heridas por proyectil por arma de fuego, con un resultado hemodinámico exitoso. Se revisó la literatura científica universal actual sobre las estrategias diagnósticas y terapéuticas que permiten establecer cuáles son las adecuadas para la atención de estos pacientes.

\section{Caso clínico}

Se trata de un paciente de sexo masculino de i8 años de edad que ingresó al servicio de urgencias de un hospital de alta complejidad especializado en trauma en Medellín (Colombia). Fue remitido desde una unidad de baja complejidad por presentar hipotensión y alteración sensorial, y heridas por proyectil de arma de fuego en la cara, el cuello (herida transfixiante), la región toracoabdominal derecha (múltiples) y el miembro superior derecho.

$\mathrm{Al}$ ingreso sus signos vitales eran: presión arterial de $\mathrm{II} 3 / 78 \mathrm{~mm} \mathrm{Hg}$, frecuencia cardíaca de I23 latidos por minuto, frecuencia respiratoria de 25 por minuto y $100 \%$ de saturación de oxígeno, después de reanimación con 2 litros de lactato de Ringer y 2 unidades de glóbulos rojos. Los resultados de los gases arteriales fueron: $\mathrm{pH}$ de 7,39; $\mathrm{pCO}_{2}$ de $33 \mathrm{~mm} \mathrm{Hg}$ y $\mathrm{pO}_{2}$ de $574 \mathrm{~mm} \mathrm{Hg}$, base exceso de $-4,4$ y lactato de $2,3 \mathrm{mmol} / \mathrm{L}$.

Por los traumas descritos, se decidió ordenar una tomografía de cuello que fue negativa para lesión traumática vascular; se encontró una herida prevertebral con trayecto transcervical y fractura lineal no desplazada del muro anterior del cuerpo vertebral $\mathrm{C}_{4}$, que ameritaba descartar una lesión no evidente del esófago o de la vía aérea.

Se practicó una tomografía toracoabdominal con contraste que mostró neumomediastino: proyectil en la arteria pulmonar del lóbulo inferior derecho, con signos de embolia pulmonar agu- 
da en sus ramas distales, proyectil en los tejidos blandos de la pared torácica posterior derecha asociado con laceración y contusión en la base pulmonar; no había neumotórax ni hemotórax; además, se apreció una laceración hepática profunda con compromiso del segmento VII, con signos de sangrado activo y hematoma subcapsular hepático contenido (figura I). Se ordenó una arteriografía que no demostró sangrado activo; también, se practicó angiotomografía y arteriografía del miembro superior derecho y no se encontró sangrado ni lesión vascular.

El paciente fue sometido a una cervicotomía derecha para explorar la herida soplante por transfixión en la zona II del cuello; se practicó sutura del esófago y toracostomía derecha, y posteriormente, se trasladó a la unidad de cuidados intensivos.
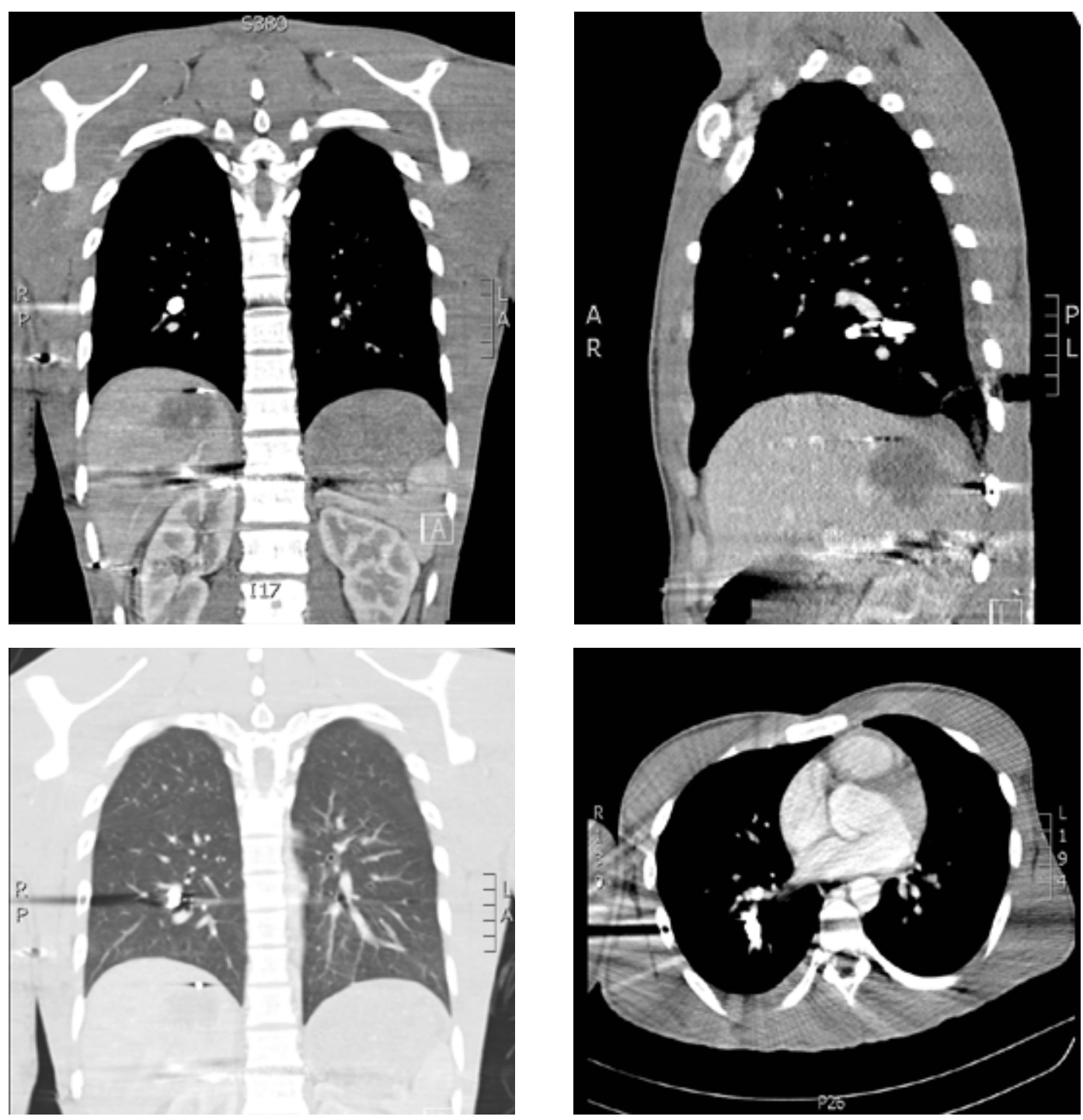

Figura 1. Angiotomografía con énfasis en el pulmón: proyectil de arma de fuego localizado en la arteria pulmonar del lóbulo inferior derecho, embolia pulmonar aguda en ramas para el lóbulo inferior derecho distal al proyectil, y proyectil en los tejidos blandos de la pared torácica posterior derecha, asociado con laceración y contusión pulmonar basal 
En forma multidisciplinaria (intervencionismo, cirugía general y cirugía de tórax) se decidió no practicar otra arteriografía por el proyectil alojado en la arteria lobar inferior derecha, pues su resultado no generaría ningún cambio en el manejo del paciente.

El paciente evolucionó satisfactoriamente y fue dado de alta a los siete días. En la cita de control ambulatorio, se observó una adecuada evolución clínica.

\section{Discusión}

El primer reporte de embolia pulmonar por herida con arma de fuego fue hecho por Thomas Davis en I934 en un niño de Io años de edad con una herida en el tórax y migración de un fragmento del proyectil hasta el ventrículo derecho ${ }^{\mathrm{I}, 2}$. Posteriormente, se han descrito otros casos por migración del proyectil en el sistema venoso y en el arterial. Hay reportes de soldados americanos con este tipo de embolia durante la segunda guerra mundial y en la guerra del Vietnam, con una incidencia cercana al $0,3 \%{ }^{3}$.

Es más frecuente entre los 20 y los 30 años de edad $(79,4 \%)$, y es más frecuente en hombres $(87,8 \%)$ que en mujeres (I2,2 \%) I. En los últimos años, su incidencia se ha debido a la violencia social civil ( $85 \%)$ más que a las guerras ( $15 \%)$. Existen otros elementos que se han asociado con la formación de embolias pulmonares, como fragmentos de hacha, elementos de acupuntura, agujas, catéteres, filtros, guías, endoprótesis (stents), válvulas, etc. ${ }^{\mathrm{I}, 4}$.

Yoon, et al., hicieron una búsqueda bibliográfica en PubMed y Google Scholar en el 20I8, y encontraron informes o series de casos descritos desde I960 hasta 20I8. En total, hallaron 54 artículos con 62 pacientes con embolias torácicas, con compromiso de aurícula derecha $(9,7 \%)$, ventrículo derecho $(54,8 \%)$, arteria pulmonar $(32,3 \%)$ y vena cava inferior intratorácica $(3,2 \%)$. Fue llamativo que solo $\mathrm{II}, 3 \%$ de los pacientes presentaron síntomas directamente relacionados con la embolia 5 .

Se ha descrito que el mecanismo del trauma se relaciona con distintos aspectos, como la energía cinética de la entrada del proyectil contra alguna estructura vascular puede sufrir alteraciones en el trayecto al disminuir dicha energía. Puede haber ruptura, aplastamiento, desgarro, abrasión o quemadura de los tejidos blandos y la migración se produce según el flujo sanguíneo.

Es más frecuente (70 \%) la migración por el sistema arterial a sitios como el cuello y las extremidades inferiores; por el sistema venoso (30\%), se localizan en las cavidades cardíacas o en las arterias pulmonares.

Se han informado casos de embolias paradójicas (I \%) a partir de fístulas arteriovenosas traumáticas o congénitas ${ }^{1,6}$. Asimismo, hay reportes de migración o embolización inmediata o tardía (hasta I4 años después) ${ }^{7}$. La trayectoria del proyectil o del objeto migratorio depende del peso, la velocidad, el tamaño, la forma, los movimientos musculares y respiratorios, la anatomía vascular, la posición del paciente en el momento del impacto, el calibre del vaso y la bifurcación vascular (inversamente proporcional) 1,5,8,9.

La sintomatología depende de la localización, la trombosis asociada, los fenómenos de la isquemia o las complicaciones hemorrágicas. Es más frecuente $(70 \%)$ que no se generen síntomas. En caso de que se generen, entre los más frecuentes se describen el dolor torácico, la disnea y la hemoptisis por la erosión vascular y el infarto pulmonar asociado ${ }^{1,8}$.

Entre las complicaciones de un proyectil de arma de fuego alojado en otras partes del cuerpo se encuentran: la isquemia, trombosis de extremidades, pseudoaneurismas, hemorragia, derrame pleural, la insuficiencia vascular, endocarditis, etc. ${ }^{10}$.

Se necesita una fuerte sospecha clínica para determinar el sitio donde se puede alojar el proyectil. En algunos casos, la sintomatología puede servir de guía, de lo contrario, solo con las imágenes y otro tipo de ayudas diagnósticas, se llega a confirmarlo ${ }^{3}$. La localización radiológica permite determinar si determinado paciente se beneficiaría o no de la extracción del proyectil y la angiotomografía de las arterias pulmonares, que es el estudio de elección ${ }^{\mathrm{I}, 3}$. Como alternativas, se puede contar -según la disponibilidad- con la ecocardiografía transesofágica o la transtorácica ${ }^{\text {I. }}$ 
En la actualidad, no existen guías de un tratamiento establecido específicamente para el manejo de un paciente con un proyectil localizado en las arterias pulmonares "I. Por esta razón, la decisión debe tomarse en forma multidisciplinaria y según con los hallazgos clínicos, el estado hemodinámico y el riesgo de las complicaciones asociadas que se han descrito.

Se ha descrito un manejo conservador cuando el paciente es asintomático, la integración del proyectil con el tejido adyacente es menor de $5 \mathrm{~mm}$, hay fragmentos libres en las cavidades cardíacas o cuando no hay riesgo de complicaciones inmediatas, como infarto pulmonar, absceso o daño bronquial ${ }^{1,12}$. La intervención quirúrgica debe considerarse en el paciente gravemente comprometido, con el proyectil localizado en arterias pulmonares principales o lobares, con gran peligro de embolia pulmonar asociada, o de localización intracardiaca con repercusión hemodinámica $u$ otro tipo de complicaciones ${ }^{13,14}$. También, existe la posibilidad de una técnica de intervencionismo radiológico, con tasas de éxito hasta del $53 \%$ en las series publicadas. Hasta el momento no hay indicaciones específicas, por lo cual la decisión debe tomarse con el equipo de trauma ${ }^{15-17}$.

En cuanto a otras conductas, a pesar de que no hay ninguna evidencia sólida que lo sugiera como una necesidad, se debe considerar la profilaxis antibiótica por el riesgo de infección asociada, principalmente endocarditis ${ }^{18,19}$. Asimismo, la anticoagulación debe considerarse por un tiempo mínimo de 12 meses, salvo ante la presencia de hemorragia significativa o estado de choque hemorrágico agudo, casos en que deberá contemporizarse ${ }^{19,20}$. Por otra parte, existe el riesgo de toxicidad por plomo, al liberarse este elemento del proyectil durante el transcurso de los años; es más frecuente el reporte de estos casos cuando el proyectil se aloja cerca de membranas sinoviales articulares ${ }^{21-23}$.

\section{Conclusiones}

La atención de estos pacientes requiere manejo por un grupo multidisciplinario que permita llegar a un diagnóstico oportuno para guiar la terapia adecuada debido a la dificultad diagnóstica y, en muchos casos, las complicaciones que se suelen presentar a corto plazo por la rápida inestabilidad hemodinámica.

La elección del manejo quirúrgico, si corresponde, debe ser individualizada en función de los síntomas y la ubicación del proyectil en el sistema vascular. Según la revisión bibliográfica y las recomendaciones de los expertos, los pacientes deben someterse a una intervención quirúrgica o endovascular cuando el proyectil se localiza en las arterias pulmonares principales o lobares y las compromete. Cuando este se localiza en las arterias segmentarias y subsegmentarias, se puede considerar un manejo conservador médico con seguimiento de imágenes diagnósticas, pues los pacientes tienden a presentar fístulas con el bronquio y vasoespasmo compensatorio, los cuales mitigarían otras complicaciones como la hipertensión pulmonar.

A la luz de la evidencia actual, no hay guías de implementación ni comparación de técnicas quirúrgicas con las intervencionistas, que permitan hacer una recomendación sólida sobre el manejo. Por esta razón, las decisiones se deben tomar con base en el grupo de trabajo multidisciplinario que evalúe al paciente.

\section{Cumplimiento de normas éticas}

Consentimiento informado. Este estudio es una revisión de historia clínica retrospectiva y, como tal, no hay necesidad de un consentimiento informado.

Conflicto de interés. Los autores declaramos no tener conflictos de interés.

Fuentes de financiación. Este estudio fue autofinanciado por los autores.

\section{Referencias}

I. Oliveira R, Fernandes D. Considerations about ballistic embolism: Experience at the João XXIII Hospital. Rev Med Minas Gerais. 20I4;24:527-34. https://doi.org/IO.5935/2238-3182.20I4OI49

2. Mattox KL, Beall AC Jr, Ennix CL, DeBakey ME. Intravascular migratory bullets. Am J Surg. 1979;137:192-5. https://doi.org/IO.IOI6/0002-96IO(79)90I43-O 
3. Shannon FL, McCroskey BL, Moore EE, Moore FA. Venous bullet embolism: Rationale for mandatory extraction. J Trauma. 1987;27:11I8-22.

https://doi.org/I0.I097/00005373-198710000-00004

4. Michelassi F, Pietrabissa A, Ferrari M, Mosca F, Vargish T, Moosa HH. Bullet emboli to the systemic and venous circulation. Surgery. 1990;107:239-45.

5. Yoon B, Grasso S, Hofmann LJ. Management of bullet emboli to the heart and great vessels. Mil Med. 2018;183:e307- e313. https://doi.org/IO.I093/milmed/usxi9I

6. Chen JJ, Mirvis SE, Shanmuganathan K. MDCT diagnosis and endovascular management of bullet embolization to the heart. Emerg Radiol. 2007;I4:I27-30. https://doi.org/IO.IOO7/sIOI40-007-0592-2

7. Nagy KK, Massad M, Fildes J, Reyes H. Missile embolization revisited: a rationale for selective management. Am Surg. 1994;60:975-9. https://doi.org/IO.I097/00005373-198710000-00004

8. Barrett NR. Foreign bodies in the cardiovascular system. Br J Surg. 1950;37:416-45. https://doi.org/IO.IO02/bjs.I80037I4804

9. Uflacker R, Lima S, Melichar AC. Intravascular foreign bodies: Percutaneous retrieval. Radiology. 1986;160:73I-5. https://doi.org/Io.II48/radiology.I60.3.37379II

Io. Cysne E, Souza EG, Freitas E, Machado E, Giambroni E, Alves LPR, et al. Embolização de projétil de arma de fogo no sistema cardiovascular. Rev Bras Cir. 1982;72:7-I2.

II. Morais D, Teixeira G, Santos G, Eduardo W, Silva J, Durante E. Peripheral arterial emboli due to bullet projectile: Diagnosis confirmed by vascular ultrasound. J Vasc Bras. 2012;II:67-72.

I2. Rehm C, Alspaugh JP, Sherman R. Bullet embolus to the right hepatic vein after a gunshot wound to the heart and its percutaneous retrieval. J Trauma. I988;28:719-20. https://doi.org/I0.I097/00005373-198805000-00035

I3. Bertoldo U, Enrichens F, Comba A, Ghiselli G, Vaccarisi S, Ferraris M. Retrograde venous bullet embolism: A rare occurrence-case report and literature review. J Trauma. 2004;57:187-92. https://doi.org/IO.IO97/oI.TA.ooooI35490.IO227.5C
I4. Wolf F, Schernthaner RE, Dirisamer A, Schoder M. Endovascular management of lost or misplaced intravascular objects: Experiences of 12 years. Cardiovasc Intervent Radiol. 2008;31:563-8. https://doi.org/I0.I007/s00270-007-920I-8

I5. Yamanari MG, Mansur MC, Kay FU, Barbosa P, Krishna $\mathrm{S}$, Buarque M. Bullet embolism of pulmonary artery: A case report. Radiol Bras. 20I4;47:128-30. https://doi.org/IO.I590/SoIOo-398420140002000I8

I6. Miller KR, Benns MV, Sciarretta JD, Harbrecht BG, Ross CB, Franklin GA, et al. The evolving management of venous bullet emboli: A case series and literature review. Injury. 20II;42:44I-6.

https://doi.org/IO.IoI6/j.injury.20I0.08.006

I7. Lu K, Gandhi S, Qureshi MA, Wright AS, Kantathut N, Noeller T. Approach to management of intravascular missile emboli: Review of the literature and case report. West J Emerg Med. 2015;16:489-96. https://doi.org/IO.58II/westjem.20I5.5.25553

18. Simpson BM, Wilson RH, Grant RE. Antibiotic therapy in gunshot wound injuries. Clin Orthop Relat Res. 2003;408:82-5.

https://doi.org/I0.I097/00003086-200303000-00008

19. Schroeder ME, Pryor HI, Chun AK, Rahbar R, Arora S, Vasin K. Retrograde migration and endovascular retrieval of venous bullet embolus. J Vasc Surg 20II;53:1113-5. https://doi.org/IO.IOI6/j.jvs.2OIO.II.046

20. Wong SS, Hockmann E, Moussa F, Bertram S. Bullet embolization from the right internal jugular vein to the right ventricle. J Cardiothorac Vasc Anesth. 2013;27:e45-6.

2I. Charris V, Guerrero A, Barrera C. Intoxicación por plomo secundaria a alojamiento de esquirlas en el cuerpo. Acta Med Colomb. 201I;36:200-3. https://doi.org/I0.36IO4/amc.20II.I450

22. Gestner JB. Intoxicación por plomo secundaria a proyectiles alojados en el cuerpo humano. Colombia Médica. 2012;43:230-4.

23. Saravi A, Torolla JL, Villafañe S, González D, Martínez M, Fernández M. Heridas de arma de fuego e intoxicación plúmbica en pediatría. Presentación de tres casos clínicos. Rev Mex Pediatría. 2017;84:196-200. 